\title{
Monitoreo del sistema eléctrico cardiovascular por medio de bici ECG
}

Monitoring of the cardiovascular electrical system by means of the ECG bike

\author{
Christian Felipe Yanez Leaño ${ }^{1}$ \\ Juan Nicolás Quiñones Romero ${ }^{2}$ \\ Ricardo Antonio Buitrago Bernal ${ }^{3}$ \\ Jorge Reynolds Pombo ${ }^{4}$ \\ Fundación Clínica Shaio
}

Recibido: 27.08.2021

Aceptado: 30.10.2021

\section{Resumen}

El conocimiento del sistema cardiovascular en el cuerpo humano es importante para comprender el funcionamiento del corazón frente a diferentes acciones o movimientos normales y rutinarios de las personas. Sin embargo, la complejidad y el costo de los equipos que exhiben este tipo de información en tiempo real, afectan la divulgación y el conocimiento que se hace necesario. Con el fin de facilitar el acceso y conocimiento, a la información de manera interactiva, se integró la tecnología de instrumentación electrónica para un monitor de

\footnotetext{
${ }^{1}$ felipe.yanezl@outlook.com

https://orcid.org/0000-0002-7969-5479

2 nicolas.quinones2107@gmail.com

https://orcid.org/0000-0002-9767-7821

${ }^{3}$ ricardo.buitrago@shaio.com

http://orcid.org/0000-0002-2602-5455

4 jorgereynolds@hotmail.com https://orcid.org/0000-0002-5490-697

Volumen 2. Número 1. Enero - Junio 2022 ISSN: 2745-0341 (En línea)
} 
electrocardiografía con el montaje de un medio de transporte muy utilizado en nuestro país; el cual es la bicicleta.

Palabras clave: Electrocardiografía, Bicicleta, Sistema Cardiovascular, corazón, electrodos.

\begin{abstract}
The knowledge of the cardiovascular system of the human body allows us to understand how the heart operates in standard and abnormal conditions. However, the complexity and cost of equipment that bring us this information in real time makes it difficult to divulge this knowledge. To facilitate access and comprehension of information in an interactive way, the electronic instrumentation for an electrocardiography monitor was integrated with the assembly on a bicycle, a way of transportation very popular in our country.
\end{abstract}

Keywords: Electrocardiography, Bicycle, Cardiovascular system, Heart, Electrodes.

\title{
Introducción
}

La electrocardiografía es un examen de diagnóstico cardiaco no invasivo que varía según el registro de potenciales eléctricos entre puntos. Según la ubicación que tienen encontramos las derivaciones bipolares, monopolares aumentadas y precordiales. Por medio de ellas se mide la actividad eléctrica del corazón representada por: La onda $P$ que traduce la despolarización auricular, el intervalo $P-R$ que hace referencia a la línea isoeléctrica, el complejo $Q R S$ para representar la despolarización ventricular y la onda T para la repolarización ventricular.

Todas estas características mencionadas anteriormente están estandarizadas para identificar posibles afecciones cardiacas en caso de que no se encuentren dentro de los rangos (O.Zavala y M. Pérez, 2004). 
Con los años, se ha implementado una técnica de electrodos alámbricos para obtener señales de electrocardiografía, con el fin de tener un monitoreo eléctrico del corazón. Esto ha permitido un gran avance en las técnicas de diagnóstico en la medicina, puesto que ha permitido la exploración de la cardiología, desvinculándose de la apropiación de una sola manera de estudio. Dicha apropiación se centraba en proporcionar la información básica, generando un diagnóstico asequible, seguro y sencillo de realizar.

Hoy en día se ha contemplado que la tecnología ha disminuido el tamaño de los electrocardiógrafos, por medio de esta implementación innovadora, la adquisición en centros de salud se ve es más oportuna que anteriormente; se reconoce la necesidad que ha surgido en frente a la comodidad del paciente para generar un dispositivo que monitoree su actividad cardiaca como hoy en día lo hace el holter. Este tipo de estudio aconseja al paciente que realice sus actividades de manera normal y cotidiana, denotamos que, a la hora de realizar la conexión de los electrodos, estos limitan las actividades dicho anteriormente "cotidianas" para el sujeto; por ende, no se puede garantizar que la toma de datos sea $100 \%$ (cien por ciento) su actividad diaria.

Los avances que ha tenido la tecnología no solamente han aportado en este tipo de equipos como el holter, ahora bien, las nuevas tecnologías de primera mano han permitido un nuevo mundo en donde el sujeto del común puede adquirir un monitoreo de su ritmo cardíaco como el BPM, como es posible mediante relojes inteligentes, pero sin embargo en el ámbito de la salud tienen limitaciones, debido a que este dato no tiene un impacto totalmente acierto para generar un diagnóstico semejante al médico (J. Bronzino y D. Peterson, 2014).

Para la adquisición del ECG y de muchas señales médicas es de vital importancia el uso del electrodo, el cual es un material conductor que al entrar en contacto con la piel capta los potenciales eléctricos, que con ello generan la señal resultante de electrocardiografía.

Existen dos tipos de electrodos, los electrodos pasivos; que son los más usados en el ámbito clínico debido a que la señal se extiende desde el material conductor hasta el equipo de 
captura; y los electrodos activos, que cuentan con un módulo de preamplificación que se da inmediatamente después del material conductor en contacto con la piel, esto con el fin de obtener una mejor calidad de la señal, antes de que se le sumen ruidos adicionales (J. Webster y J. Clark, 2010).

\section{Desarrollo}

Al contemplar que el electrocardiograma digitalizado es útil y necesario frente a la innovación tecnológica en la salud, es evidente la responsabilidad que hay de preservar la enseñanza en el creciente conocimiento de su lectura como la innovación para su accesibilidad. Frente a esa idea surgieron necesidades como la comodidad y funcionalidad de un monitoreo del sistema eléctrico cardiovascular que cumpla con dichas especificaciones.

Se llegó a comprender que hay oportunidades de aprendizaje y mejoras sociales, como surgieron en medio de este proceso de búsqueda y estudio; se contempló la idea de utilizar un artefacto del común como prioridad para el análisis eléctrico y así poder aportar no solo a una nueva tecnología, sino que hiciera parte de un cambio en el ecosistema al implementarlo.

En base a lo anterior se planteó el monitoreo como un nuevo proyecto que incorpora el electrocardiograma en un utensilio cotidiano como lo es la bicicleta, esto con el fin de llegar con mayor facilidad a los usuarios y generar conciencia del funcionamiento en tiempo real de su propio sistema cardiovascular.

Se le otorga el nombre de BICI ECG, el cual consta con un juego de electrodos de placa metálica instalados en el manubrio de una bicicleta comercial para obtener la señal biológica; esto es posible al afirmar los extremos del manubrio con las palmas (M. Guardiola, 2020).

Se incorpora igualmente una ayuda visual que simboliza la contracción en tiempo real del corazón del paciente por medio de un haz de luz en un artefacto diseñado como un corazón humano con una medida amplia que se ilustró y construyó en 3D, imprimiendo dicho diseño en una impresora 3D como se ve en la figura 1, que se sincroniza con el complejo QRS de la 
señal electrocardiográfica y un buzzer que se encarga de generar el sonido característico de los monitores de electrocardiografía.

Figura 1. Montaje del BICI ECG en funcionamiento.

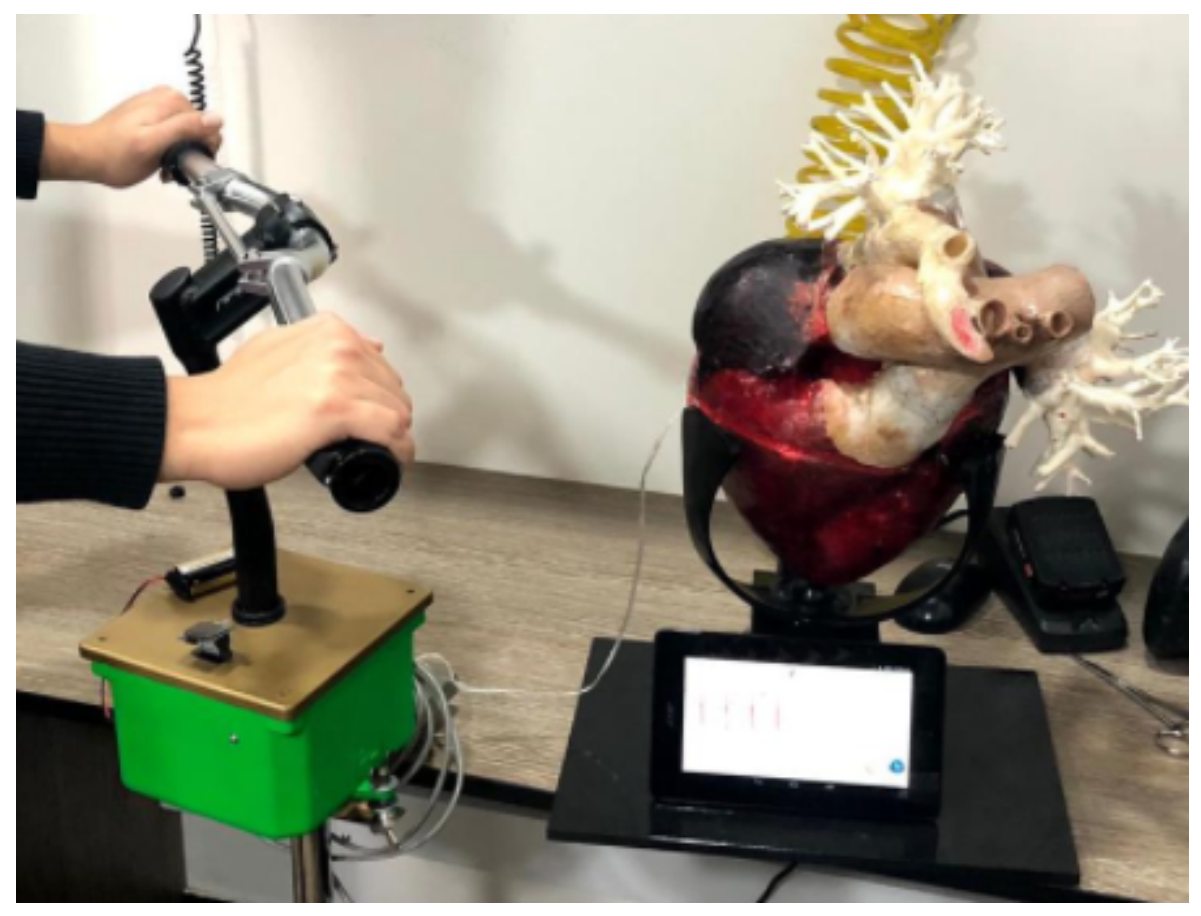

Gracias a la implementación visual el bioinstrumento (BICI ECG) tiene una comunicación inalámbrica, la cual transmite la información de la onda completa de electrocardiografía por medio de bluetooth para visualizar detalladamente las ondas que tiene el paciente en tiempo real. Al construir este proyecto surgieron mejoras en el proceso como la adquisición de una aplicación en Android la cual permite la visualización de las señales como se puede ver en la Figura 2. 
Figura 2. Pantalla de tablet receptora bluetooth de la señal de electrocardiografía.

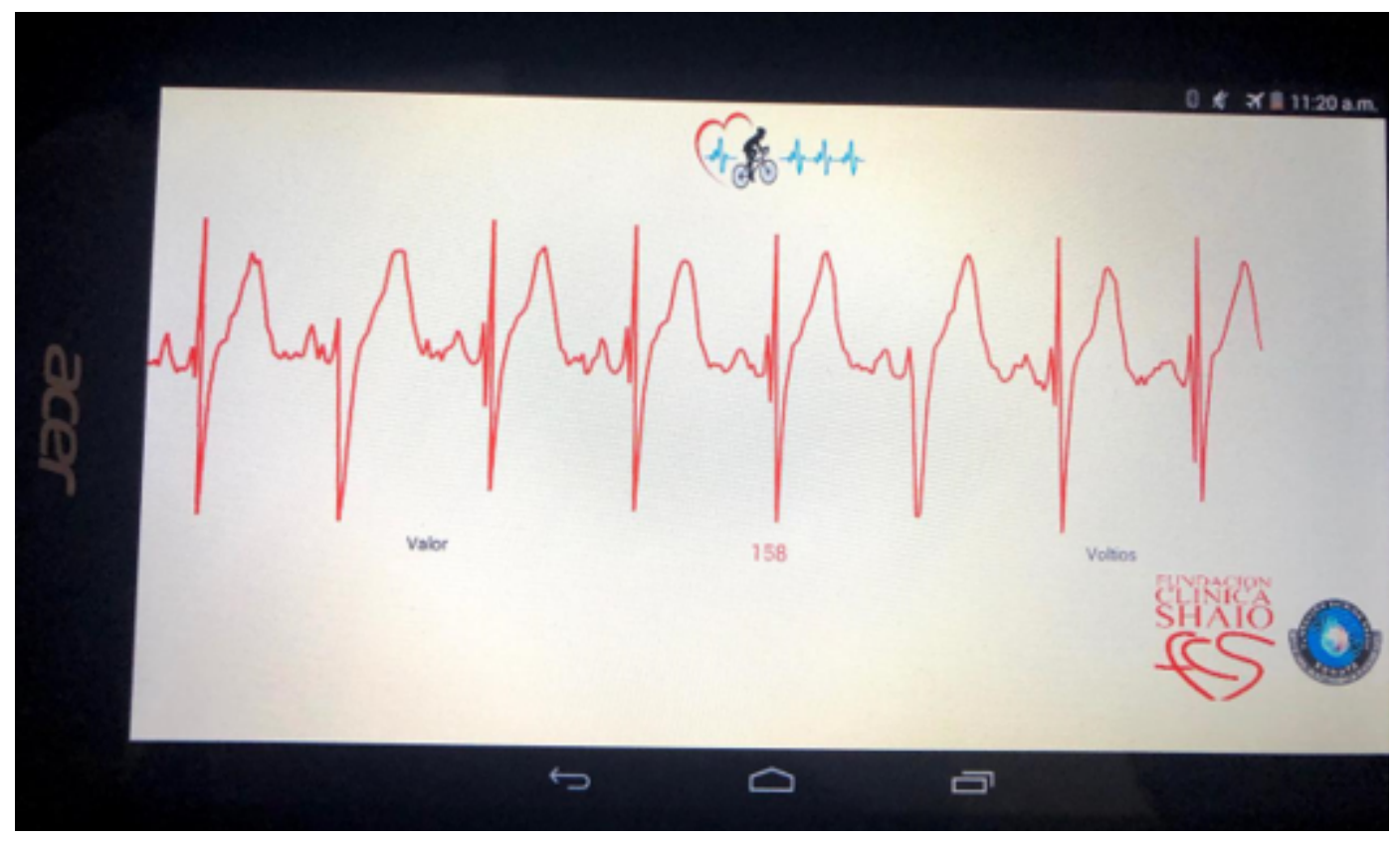

Así mismo el bioinstrumento (BICI ECG) tiene una comunicación inalámbrica la cual transmite la información de la onda completa de electrocardiografía por medio de bluetooth para ver con más detalle todas las ondas que tiene el paciente en tiempo real. Adicionalmente para la visualización de la información se desarrolló una aplicación android como se puede ver en la Figura 2.

\section{Conclusiones}

El BICI ECG genera una experiencia de conocimiento del sistema cardiovascular a partir de un sistema envolvente que mezcla el instrumento de la bicicleta con un corazón impreso en 3D que tiene una luz y sonido sincronizado con la contracción del corazón en tiempo real del usuario de la bicicleta. Este instrumento permite generar una facilidad en la accesibilidad del conocimiento y de equipos que permiten ver el funcionamiento del corazón de los pacientes y así promulgar la educación y conocimiento propio del sistema cardiovascular. 


\section{Referencias}

Bronzino, J. D. and Peterson, D. R. (2014). The Biomedical Engineering Handbook, Third Edition - 3 Volume Set: Biomedical Engineering Fundamentals, 2 edition. Boca Raton: CRC Press.

M. Guardiola, MÁ. (2020). Localización automática de electrodos de ECG para Imagen Electrocardiográfica utilizando Identificación de Patrones. http://hdl.handle.net/10251/152392

Webster J. G. and Clark, J. W. (2010). Medical instrumentation: application and design. Hoboken, New Jersey: John Wiley \& Sons.

Zavala Zamora, O. D. y Perez de Juan, M. A. (2004). LIBRO ELECTROCARDIOGRAFIA CLINICA, Segunda edición ELSEVIER España S.A. 
REVISTA INTERNACIONAL DE PEDAGOGÍA E INNOVACIÓN EDUCATIVA

Volumen 2. Número 1. Enero - Junio 2022 ISSN: 2745-0341 (En línea) 